\title{
Nephrotoxicity and Efficacy Assessment of Polymyxin B Use in Renal Transplant Patients
}

\author{
Yu-Xin Wen ${ }^{1,2, *}$, Qiang $\mathrm{Qu}^{3,4, *}$, Wen-Ming Long ${ }^{5}$, Yue Luo ${ }^{6}$, Hai-Hui Zhuang' ${ }^{\prime}$ Xin-Qi Teng' ${ }^{\prime}$ Jian Qu (i) ${ }^{\prime}$
}

'Department of Pharmacy, The Second Xiangya Hospital, Central South University; Institute of Clinical Pharmacy, Central South University, Changsha, 4 I00I I, People's Republic of China; ${ }^{2}$ Department of Pharmacy, Lixian People's Hospital in Hunan, Lixian, 4I5500, People's Republic of China; ${ }^{3}$ Department of Pharmacy, Xiangya Hospital, Central South University, Changsha, 410078, People's Republic of China; ${ }^{4}$ National Clinical Research Center for Geriatric Disorders, Xiangya Hospital, Central South University, Changsha, 410078, People’s Republic of China; ${ }^{5}$ Department of Pharmacy, Jingzhou District, Second People's Hospital of Huaihua City, Huaihua, 418400, People's Republic of China; ' ${ }^{6}$ epartment of Pharmacy, The People's Hospital of Liuyang, Liuyang, 4 I 0300 , People's Republic of China

*These authors contributed equally to this work

Correspondence: Jian Qu, Department of Pharmacy, The Second Xiangya Hospital, Central South University; Institute of Clinical Pharmacy, Central South University, No. 139 Middle Renmin Road, Changsha, 4I00II, People’s Republic of China, Tel +86-I5973I906I4, Fax +86-73I-85292072,

Email qujianstanley@csu.edu.cn

Purpose: This study investigates the nephrotoxicity and efficacy assessment of polymyxin B (PMB) use in renal transplant patients. Patients and Methods: This retrospective study included adult ( $>18$ years of age) renal transplant patients who received PMB intravenous drip for more than 72 hours. Efficacy assessment of PMB included clinical treatment efficacy, microbiological efficacy at the end of PMB treatment, and in-hospital all-cause mortality. Nephrotoxicity of PMB was evaluated for further group comparison.

Results: We enrolled 235 renal transplant patients in our study. After PMB treatment, 45 patients occurred PMB-nephrotoxicity, and the nephrotoxicity rate was $19.15 \%$. Among them, 44 patients were RIFLE R stage, and one patient was RIFLE I stage. The dose of PMB used in patients was $40.0(40.0-50.0) \mathrm{mg} \mathrm{q12h}$ with a loading dose of $41.8 \pm 9.8 \mathrm{mg}$. Multivariate logistic regression analysis showed that ICU admission, vasoactive agents, aminoglycosides, creatinine clearance rate before PMB use, and mean total hospital stay were independent risk factors of PMB-nephrotoxicity in kidney transplant patients. The clinical effective rate was $97.9 \%$, and the microbiological clean rate was $66.7 \%$.

Conclusion: Our study demonstrated that PMB low dose regimens might achieve good efficacy and less nephrotoxicity in renal transplant patients. We should evaluate the severity of the infection and renal function of patients, avoid the combined use of other nephrotoxic drugs, and minimize the course of use to reduce the occurrence of PMB-nephrotoxicity.

Keywords: polymyxin B, nephrotoxicity, renal transplant patients, adverse reactions

\section{Introduction}

Polymyxins are old antimicrobials discovered in the 1940 s, and clinical use started in the late 1960 s. ${ }^{1}$ Due to the emerging of multidrug-resistant (MDR) Gram-negative bacteria infection, currently available antibiotics are limited. Polymyxins, including colistin and polymyxin B (PMB), were the last resort to defense against the Carbapenem-resistant gram-negative bacteria (CR-GNB) infection. ${ }^{2} \mathrm{PMB}$ is administered to patients in its active form, while colistin is used in the form of its inactive prodrug colistimethate (CMS), which is required conversion to colistin in vivo. ${ }^{3}$

Although PMB as the "last-line" antibiotics to CR-GNB infection showed powerful and broad-spectrum antinegative bacteria, PMB-associated nephrotoxicity limits the optimization of its dose, thus limiting the increase of its efficacy. ${ }^{4}$ Acute kidney injury (AKI) occurs in a substantial proportion of patients receiving PMB and is the major doselimiting adverse effect of the polymyxins. ${ }^{3,5}$ PMB's pharmacokinetics and pharmacodynamic properties are limited. ${ }^{6,7}$ There is a paucity of information regarding its clinical use, especially in special populations, such as renal transplant patients. $^{8-10}$ 
A recent study about population pharmacokinetics of PMB and dosage optimization with Monte Carlo simulation found that a $75 \mathrm{mg}$ loading dose with a $50 \mathrm{mg}$ maintenance dose was a better option to achieve an optimal therapeutic effect (minimum inhibitory concentration $\leq 1 \mathrm{mg} / \mathrm{L}$ ) and to reduce the incidence of nephrotoxicity for patients with renal impairments. ${ }^{8}$ In a retrospective study in 92 solid organ transplant patients treated with polymyxin (polymyxins B and E), the nephrotoxicity rate was $32.6 \%$. The duration of polymyxin was associated with significant renal function dysfunction. ${ }^{9}$ In clinical, renal transplant patients have high-grade antibiotic exposure, prolonged hospitalization, and the immune-suppression state. Therefore, they are at high risk of CR-GNB infection. ${ }^{11}$ At the same time, the renal dysfunction of renal transplant patients limited the use of PMB, which has a high nephrotoxicity rate. Previous studies demonstrated the influencing factors of PMB-associated nephrotoxicity include malignancy, co-infection with other microorganisms, being elderly, the high daily dose of PMB, having underlying diseases such as diabetes, and using concomitant nephrotoxic drugs. Whether these factors also influence the incidence of nephrotoxicity in renal transplant patients still needs further studies.

Moreover, in clinical practice, clinicians usually did not follow the recommended dose according to international guidelines ${ }^{12}$ because they were worried about the effect of PMB on the renal function of transplant patients. In the real world, the impact of PMB at doses lower than recommended by the guideline on infection and renal function in kidney transplant patients are worth further exploring. This study aims to investigate the nephrotoxicity and efficacy assessment of PMB use in renal transplant patients.

\section{Patients and Methods}

\section{Ethics}

The Ethics Committees of the Second Xiangya Hospital of Central South University (LYF-2020021) approved our retrospective study. We followed the ethical standards laid down in the 1964 Declaration of Helsinki, and informed consent was waived because of non-interventional in our research. All organs were donated voluntarily with written informed consent, and that this was conducted in accordance with the Declaration of Istanbul.

\section{Patients}

This retrospective cohort study was carried out in the Second Xiangya Hospital of Central South University, a 3500-bed grade-A hospital, from May 1st, 2018, to July 31st, 2021. We included adult ( $>18$ years of age) renal transplant patients who received PMB (Shanghai Number 1 Biochemical \& Pharmaceuticals, Shanghai, China) intravenous drip for more than 72 hours. Exclusion criteria were (i) not renal transplant patients; (ii) PMB use less than three days; (iii) $<18$ years old or pregnant; (iii i) incomplete clinical data.

\section{Clinical Data Collection}

Clinical data extracted from patients' electronic records included demographics including recipient age, gender, comorbidities, type of infections and pathogenic bacteria, infection sites, concomitant nephrotoxic agents, details of PMB use (loading dose, daily dose based on total body weight, duration of treatment, and cumulative PMB dose), kidney status (creatinine and creatinine clearance rate (CCR) before PMB use, creatinine, and CCR after PMB treatment, CRRT or hemodialysis), and immunosuppressant at time of PMB use.

\section{Microbiology}

Analytical instruments by the broth microdilution method were used to test the sensitivity of bacteria to antibacterial drugs. Minimum inhibitory concentration (MIC) was determined by a VITEK ${ }^{\circledR} 2$ system (bioMérieux, Marcy-l'Étoile, France) based on the recommendations of the European Committee on Antimicrobial Susceptibility Testing (EUCAST) and the Clinical and Laboratory Standards Institute (CLSI) (Dalyan et al, 2018; (CLSI), 2020). Carbapenem resistance was defined as strains isolated with non-susceptibility ( $M I C \geq 4 \mathrm{mg} / \mathrm{L}$ ) to imipenem or meropenem, according to the 2019 interpretative criteria by the CLSI ((CLSI), 2020). Polymyxins and tigecycline resistance were MIC $>2 \mathrm{mg} / \mathrm{L}$ as per the EUCAST guideline (http://www.eucast.org/clinical breakpoints) (Dalyan et al, 2018). 


\section{Definitions}

PMB-nephrotoxicity was defined as the incidence of acute kidney injury (AKI) after PMB use. According to the Kidney Disease: Improving Global Outcomes (KDIGO) criteria, ${ }^{13}$ In patients with normal renal function (GFR $\geq 60 \mathrm{~mL} / \mathrm{min} /$ $\left.1.73 \mathrm{~m}^{2}\right)$, AKI was defined as an increase in serum creatinine by $26.4 \mathrm{umol} / \mathrm{L}(0.3 \mathrm{mg} / \mathrm{dl})$ within 48 hours or a $50 \%$ increase in serum creatinine from baseline within seven days, or as a decline in renal function that resulted in the need for renal replacement therapy (ie, intermittent hemodialysis or continuous venovenous hemofiltration). In patients with preexisting renal dysfunction, renal failure was defined as an increase of 50\% over the baseline creatinine level, as a reduction in the calculated creatinine clearance of $50 \%$ relative to the value at antibiotic therapy initiation, or as a decline in renal function that resulted in the need for renal replacement therapy. ${ }^{9}$

To analysis the severity of AKI, we also used KDIGO to define the stage of AKI as Risk (R), Injury (I), Failure (F). AKI phased R, I, and F were defined as the blood creatinine levels within 48 hours of discontinuation of PMB were 1.5-1.9 times, 2.0-2.9 times, and three times higher than the baseline, respectively. ${ }^{13}$ Baseline creatinine was the last creatinine obtained before antibiotic initiation (This value must have been obtained within 48 hours before antibiotic initiation). Follow-up continued until 48 hours after the last dose.

Efficacy Assessment of PMB included clinical treatment efficacy, microbiological efficacy at the end of PMB treatment, and in-hospital all-cause mortality. Clinical efficacy of PMB targeted therapy was defined as meeting all of the following conditions: (i) being hemodynamically stable without the need for vasopressors; (ii) body temperature $<37.5^{\circ} \mathrm{C}$ within 72 hours; (iii) improved clinical and microbiologic parameters. Clinical efficacy of empirical therapy was defined as the body temperature $<37.5^{\circ} \mathrm{C}$ within 72 hours, being hemodynamically stable without the need for vasopressors, and the improved inflammatory index. Clinical treatment failure was defined as patients who failed to meet any clinical efficacy criteria, stopped treatment with low blood pressure, or died in hospital. ${ }^{14}$ Microbiological efficacy was defined as the clearance of strains within the total course of PMB, demonstrated by negative microbial culture results of samples from the same site after PMB treatment. In-hospital all-cause mortality was defined as all-cause death or stopped treatments with low blood pressure.

\section{Statistical Analysis}

We used SPSS 25 (IBM, Armonk, NY, USA) to analyze the data. Continuous data were presented using mean and standard deviation (SD) or median and interquartile ranges (IQR). Count data were presented as quantity and percentage. Univariate analysis and binary logistic regression were used to compare patients with and without PMB-associated nephrotoxicity. The factors with a $\mathrm{P}$ value of 0.1 by univariate analysis were included in the binary logistic regression model. A final two-tailed p-value less than 0.05 was considered significant.

\section{Results}

\section{Patients' Demographic Data}

According to the inclusion and exclusion criteria, we enrolled 235 renal transplant patients treated with PMB in our study. After the assessment of nephrotoxicity, 45 patients occurred PMB-nephrotoxicity. Among them, 44 patients were RIFLE R stage, and one patient was RIFLE I stage. There was no nephrotoxicity in 190 patients after PMB use. Table 1 shows the demographic and clinical characteristics of total enrolled patients and compares positive and negative PMBnephrotoxicity patients. The median recipient age of the patients was $41.1 \pm 12.9$ years, and $27.2 \%$ of patients were women. The mean total hospital stay days were 26.0 (17.0-40.0) days, and $11.5 \%$ of patients were admitted to ICU. The total hospital stay in patients with nephrotoxicity was longer than that in patients without nephrotoxicity. A higher proportion of patients with nephrotoxicity were admitted to ICU patients compared with patients without nephrotoxicity. There were $10.6 \%$ of patients with multiple-site infections, and $51.1 \%$ were single-site infections. Mechanical ventilation and vasoactive agents were more frequent happened in PMB-nephrotoxicity patients. Moreover, $38.3 \%$ of patients had no definite infection site or culture results. Lung infection was the main infection site, accounting for $46.8 \%$. Lung infection portions were higher in patients with PMB-nephrotoxicity than patients without nephrotoxicity $(66.7 \%$ vs $42.1 \%$, $\mathrm{p}=0.003$ ). The percentage of liver disease is higher in patients with PMB-nephrotoxicity than patients without 
Table I Demographics and Clinical Characteristics of the Study Cohort

\begin{tabular}{|c|c|c|c|c|}
\hline Variables & $\begin{array}{l}\text { Total Patients } \\
(\mathrm{N}=235)\end{array}$ & $\begin{array}{l}\text { PMB-Nephrotoxicity }(+) \\
(\mathrm{N}=45)\end{array}$ & $\begin{array}{l}\text { PMB-Nephrotoxicity (-) } \\
(\mathrm{N}=190)\end{array}$ & P-value* \\
\hline Mean recipient age (year) & $41.1 \pm 12.9$ & $41.9 \pm 14.3$ & $40.9 \pm 12.5$ & 0.644 \\
\hline Gender (Male/Female) & $171 / 64$ & $38 / 7$ & 133/57 & 0.050 \\
\hline Mean total hospital stay (days) & $26(17.0-40.0)$ & $42(29.5-102.0)$ & $24(16.00-32.3)$ & $<0.001$ \\
\hline ICU admission & $27(11.5 \%)$ & $14(31.1 \%)$ & $13(6.8 \%)$ & $<0.001$ \\
\hline Mechanical ventilation & $13(5.5 \%)$ & $10(22.2 \%)$ & $3(1.6 \%)$ & $<0.001$ \\
\hline Vasoactive agents & $58(24.7 \%)$ & $20(44.4 \%)$ & $38(20.0 \%)$ & 0.001 \\
\hline The time after renal transplantation (month) & $0.6(0.0-7.0)$ & $0.7(0.3-7.8)$ & $0.4(0.0-6.6)$ & 0.145 \\
\hline Infection site & & & & 0.061 \\
\hline Multiple & $25(10.6 \%)$ & $4(8.9 \%)$ & $21(11.1 \%)$ & \\
\hline Single & $120(5 \mid .1 \%)$ & $30(66.7 \%)$ & $90(47.7 \%)$ & \\
\hline Unclear & $90(38.3 \%)$ & II (24.4\%) & $79(41.6 \%)$ & \\
\hline \multicolumn{5}{|l|}{ Source of infection } \\
\hline Respiratory tract & $110(46.8 \%)$ & $30(66.7 \%)$ & $80(42.1 \%)$ & 0.003 \\
\hline Blood & $15(6.4 \%)$ & $2(4.4 \%)$ & $13(6.8 \%)$ & 0.801 \\
\hline Urinary tract & $33(14.0 \%)$ & $4(8.9 \%)$ & $29(15.3 \%)$ & 0.385 \\
\hline Abdominal & $4(1.7 \%)$ & $\mathrm{I}(2.2 \%)$ & $3(1.6 \%)$ & 0.575 \\
\hline \multicolumn{5}{|l|}{ Comorbidities } \\
\hline Cardiovascular and cerebrovascular diseases & $80(34.0 \%)$ & $20(44.4 \%)$ & $60(31.6 \%)$ & 0.101 \\
\hline Respiratory disease & $6(2.6 \%)$ & $0(0.0 \%)$ & $6(3.2 \%)$ & 0.495 \\
\hline Diabetes mellitus & $37(15.7 \%)$ & $8(17.8 \%)$ & $29(15.3 \%)$ & 0.677 \\
\hline Diseases of digestive system & $50(21.3 \%)$ & II (24.4\%) & $39(20.5 \%)$ & 0.564 \\
\hline Nutritional disease & $18(7.7 \%)$ & $6(33.3 \%)$ & $12(66.7 \%)$ & 0.111 \\
\hline Liver disease & $30(12.7 \%)$ & II (24.4\%) & $19(10.0 \%)$ & 0.009 \\
\hline \multicolumn{5}{|l|}{ Types of GR-GNB strains } \\
\hline Acinetobacter baumannii & $5(2.1 \%)$ & $\mathrm{I}(2.2 \%)$ & $4(2.1 \%)$ & 1.000 \\
\hline Klebsiella pneumoniae & $17(7.2 \%)$ & $5(11.1 \%)$ & $12(6.3 \%)$ & 0.264 \\
\hline Pseudomonas aeruginosa & $6(2.6 \%)$ & $\mathrm{I}(2.2 \%)$ & $5(2.6 \%)$ & 1.000 \\
\hline \multicolumn{5}{|l|}{ Immunosuppressant at time of PMB use } \\
\hline Prednisone & $209(88.9 \%)$ & $38(84.4 \%)$ & $|7|(81.8 \%)$ & 0.285 \\
\hline Dexamethasone & $32(13.6 \%)$ & $15(33.3 \%)$ & $17(8.9 \%)$ & $<0.001$ \\
\hline Tacrolimus & $177(75.3 \%)$ & $25(55.6 \%)$ & $152(80.0 \%)$ & 0.001 \\
\hline Mycophenolate mofetil & $161(68.5 \%)$ & $22(48.9 \%)$ & $139(73.2 \%)$ & 0.002 \\
\hline Cyclosporine & $25(10.6 \%)$ & $8(17.8 \%)$ & $17(8.9 \%)$ & 0.084 \\
\hline \multicolumn{5}{|l|}{ Outcome } \\
\hline Hospital mortality & $4(1.7 \%)$ & $2(4.4 \%)$ & $2(1.1 \%)$ & 0.166 \\
\hline Microbiological cure & $32 / 48(66.7 \%)$ & $8(61.5 \%)$ & $24(68.6 \%)$ & 0.646 \\
\hline Clinical cure & $230(97.9 \%)$ & $42(93.3 \%)$ & $188(98.9 \%)$ & 0.019 \\
\hline
\end{tabular}

Notes: Data were present as Mean \pm standard deviation, No (\%), or Median (IQR).*P value were shown as PMB-Nephrotoxicity (+) vs PMB-Nephrotoxicity (-). Bold font indicates data with significant differences.

Abbreviations: ICU, intensive care unit; APACHE II, Acute Physiology and Chronic Health Evaluation II; TMP-SMZ, trimethoprim/sulfamethoxazole; NSAIDS, nonsteroidal anti-inflammatory drugs; PMB, polymyxin B; CR-GNB, carbapenem-resistant Gram-negative bacteria; CRRT, continuous renal replacement therapy.

nephrotoxicity $(20.4 \%$ vs $10.0 \%, \mathrm{p}=0.009)$. The distributions of immunosuppressant use such as dexamethasone, tacrolimus, and mycophenolate mofetil differed between the two groups. Clinical efficacy in patients without PMBnephrotoxicity was higher than in patients with PMB-nephrotoxicity $(98.9 \%$ vs $93.3 \%, \mathrm{p}=0.019)$. The susceptibility of PMB and tigecycline of isolated CR-GNB strains from patients are shown in Table 2. Moreover, we evaluated the recovery of renal function before discharge after PMB withdrawal in patients with PMB-nephrotoxicity and there were 27 (27/45, 60\%) patients' CCR increased and they had partial recovery of renal function.

\section{Kidney Status and Nephrotoxic Agents Use in Kidney Transplant Patients}

The portions of concomitant nephrotoxic agents used, such as aminoglycosides, amphotericin B fluorocytosine, and torasemide $(>10 \mathrm{mg} /$ day), were higher in patients with PMB-nephrotoxicity than patients without PMB-nephrotoxicity 
Table 2 The PMB and TGC Susceptibility of CR-GNB

\begin{tabular}{|c|c|c|c|c|c|c|c|}
\hline \multirow[t]{2}{*}{ Type of CR-GNB } & \multirow[t]{2}{*}{ Numbers of Strains } & \multicolumn{4}{|c|}{ PMB MIC } & \multicolumn{2}{|c|}{ TGC MIC } \\
\hline & & $\leqq 0.5$ & $\leqq 1$ & I & 2 & $\leqq 1$ & 4 \\
\hline CRKP & 17 & 2 & 12 & 3 & 0 & 5 & 12 \\
\hline CRAB & 5 & 3 & 1 & 1 & 0 & 2 & 3 \\
\hline CRPA & 6 & 0 & 1 & 4 & 1 & - & - \\
\hline
\end{tabular}

Abbreviations: PMB, polymyxin B; TGC, tigecycline; CR-GNB, carbapenem-resistant Gram-negative bacteria; MIC, minimum inhibitory concentration; CRKP, carbapenemresistant Klebsiella pneumonia; CRPA, carbapenem-resistant Pseudomonas aeruginosa; CRAB, carbapenem-resistant Acinetobacter baumannii.

(Table 3). The loading and maintenance dose of PMB used in patients was relatively small, $41.8 \pm 9.8 \mathrm{mg}$ and 40.0 (40.050.0) $\mathrm{mg} \mathrm{q} 12 \mathrm{~h}$, respectively. The cumulative dose of PMB was higher in patients with PMB-nephrotoxicity than patients without nephrotoxicity (880.0 (520.0-1110.0) $\mathrm{mg}$ vs $640.0(425.0-900.0), \mathrm{p}=0.006)$. The treatment duration of PMB in all patients was 8.0 (6.0-11.0) days and more extended in patients with PMB-nephrotoxicity than that in patients without nephrotoxicity (11.0 (7.0-15.0) days vs 8.0 (5.8-11.0) days, $\mathrm{p}=0.003)$. Creatinine before PMB use was higher in the PMB-nephrotoxicity group than without the PMB-nephrotoxicity group. The portion of CRRT and hemodialysis were also higher in the PMB-nephrotoxicity group than the without PMB-nephrotoxicity group.

\section{Risk Factors for PMB-Nephrotoxicity in Kidney Transplant Patients}

Multivariate logistic regression analysis showed that ICU admission (OR=4.311 (1.013-18.351), $\mathrm{P}=0.048)$, vasoactive agents $(\mathrm{OR}=2.779$ (1.034-7.465), $\mathrm{P}=0.043)$, aminoglycosides ( $\mathrm{OR}=19.793$ (1.744-224.604), $\mathrm{P}=0.016), \mathrm{CCR}$ before $\mathrm{PMB}$ use $(\mathrm{OR}=0.966(0.937-0.995), \mathrm{P}=0.023)$, and mean total hospital stay $(\mathrm{OR}=1.029(1.013-1.004), \mathrm{P}<0.001)$ were independent risk factors of PMB-nephrotoxicity in kidney transplant patients (Table 4).

Table 3 Kidney Status and Nephrotoxic Agents Used in Kidney Transplant Patients

\begin{tabular}{|c|c|c|c|c|}
\hline Variables & $\begin{array}{l}\text { Total Patients } \\
(\mathrm{N}=235)\end{array}$ & $\begin{array}{l}\text { PMB-Nephrotoxicity }(+) \\
(\mathrm{N}=45)\end{array}$ & $\begin{array}{l}\text { PMB-Nephrotoxicity (-) } \\
(\mathrm{N}=190)\end{array}$ & P-value \\
\hline \multicolumn{5}{|l|}{ Concomitant nephrotoxic agents } \\
\hline Aminoglycosides & $6(2.6 \%)$ & $4(8.9 \%)$ & $2(1.1 \%)$ & 0.013 \\
\hline Amphotericin B & $24(10.2 \%)$ & $10(22.2 \%)$ & $14(7.4 \%)$ & 0.003 \\
\hline TMP-SMZ & $64(27.2 \%)$ & $17(37.8 \%)$ & $44(23.2 \%)$ & 0.044 \\
\hline Fluorocytosine & $9(3.8 \%)$ & $4(8.9 \%)$ & $5(2.6 \%)$ & 0.125 \\
\hline Furosemide (>20mg/day) & $55(23.4 \%)$ & $12(26.7 \%)$ & $43(22.6 \%)$ & 0.705 \\
\hline Torasemide (>10mg/day) & $15(6.4 \%)$ & $7(15.6 \%)$ & $8(4.2 \%)$ & 0.005 \\
\hline NSAIDS & $3(1.3 \%)$ & $\mathrm{I}(2.2 \%)$ & $2(1.1 \%)$ & 0.473 \\
\hline \multicolumn{5}{|l|}{ PMB use } \\
\hline Empiric treatment/Target treatment & $187 / 48$ & $33 / 12$ & $154 / 36$ & 0.248 \\
\hline Using loading dose & $23(9.8 \%)$ & $7(15.6 \%)$ & $16(8.4 \%)$ & 0.148 \\
\hline Loading dose (mg) & $41.8 \pm 9.8$ & $43.3 \pm 11.6$ & $41.4 \pm 9.3$ & 0.240 \\
\hline Maintenance dose $q / 2 \mathrm{~h}(\mathrm{mg})$ & $40.0(40.0-50.0)$ & $40.0(40.0-50.0)$ & $40.0(40.0-50.0)$ & 0.752 \\
\hline Maintenance dose in $\mathrm{mg} / \mathrm{kg}(q / 2 \mathrm{~h})$ & $0.7 \pm 0.2$ & $0.7 \pm 0.2$ & $0.7 \pm 0.2$ & 0.902 \\
\hline Minimum maintenance dose $(>1.25 \mathrm{mg} / \mathrm{kq}, \mathrm{q} / 2 \mathrm{~h})$ & $3(1.3 \%)$ & $0(0 \%)$ & $3(1.6 \%)$ & 1.000 \\
\hline Cumulative dose $(\mathrm{mg})$ & $640.0(450.0-960.0)$ & $880.0(520.0-1110.0)$ & $640.0(425.0-900.0)$ & 0.006 \\
\hline Treatment duration time (days) & $8.0(6.0-11.0)$ & $11.0(7.0-15.0)$ & $8.0(5.8-11.0)$ & 0.003 \\
\hline \multicolumn{5}{|l|}{ Kidney status } \\
\hline Creatinine before PMB use (umol/L) & $429.2 \pm 368.4$ & $526.1 \pm 366.7$ & $406.2 \pm 366.1$ & 0.049 \\
\hline CRRT & $10(4.3 \%)$ & $10(22.2 \%)$ & $0(0 \%)$ & $<0.001$ \\
\hline Hemodialysis & $44(18.7 \%)$ & $39(86.7 \%)$ & $5(2.6 \%)$ & $<0.001$ \\
\hline
\end{tabular}

Notes: Data were present as Mean \pm standard deviation, No.(\%), or Median (IQR). Bold font indicates data with significant differences.

Abbreviations: AKI, acute kidney injury; ICU, intensive care unit; APACHE II, Acute Physiology and Chronic Health Evaluation II; DM, diabetes mellitus; CR-GNB, carbapenem-resistant Gram-negative bacteria; TMP-SMZ, trimethoprim/sulfamethoxazole; NSAIDs, nonsteroidal anti-inflammatory drugs; PMB, polymyxin B; CRRT, continuous renal replacement therapy. 
Table 4 Multivariate Logistic Regression of PMB-Nephrotoxicity in Kidney Transplant Patients

\begin{tabular}{|c|c|c|c|}
\hline Variable & Adjusted OR & $95 \% \mathrm{Cl}$ & P-value \\
\hline Gender (Male/Female) & 0.416 & $0.122-1.420$ & 0.161 \\
\hline Mean total hospital stay (days) & 1.029 & $1.013-1.044$ & $<0.001$ \\
\hline ICU admission & 4.311 & $|.0| 3-|8.35|$ & 0.048 \\
\hline Mechanical ventilation & 1.344 & $0.129-14.047$ & 0.805 \\
\hline Vasoactive agents & 2.779 & $1.034-7.465$ & 0.043 \\
\hline Respiratory tract & 0.958 & $0.287-3.205$ & 0.945 \\
\hline Liver disease & 3.118 & $0.969-10.028$ & 0.056 \\
\hline Aminoglycosides & 19.793 & $1.744-224.604$ & 0.016 \\
\hline Amphotericin B & 2.611 & $0.425-16.058$ & 0.300 \\
\hline TMP-SMZ & 0.818 & $0.224-2.985$ & 0.761 \\
\hline Furosemide ( $>20 \mathrm{mg} /$ day $)$ & 0.600 & $0.200-1.796$ & 0.361 \\
\hline Cumulative dose (mg) & 0.999 & $0.996-1.001$ & 0.375 \\
\hline Treatment duration time (days) & 1.199 & $0.94 I-I .527$ & 0.141 \\
\hline Dexamethasone & 2.486 & $0.744-8.312$ & 0.139 \\
\hline Tacrolimus & 0.636 & $0.15 \mathrm{I}-2.674$ & 0.537 \\
\hline Mycophenolate mofetil & 1.409 & $0.369-5.376$ & 0.616 \\
\hline CCR before PMB use $\left(\mathrm{mL} / \mathrm{min} .1 .73 \mathrm{~m}^{2}\right)$ & 0.966 & $0.937-0.995$ & 0.023 \\
\hline
\end{tabular}

Note: Bold font indicates data with significant differences.

Abbreviations: ICU, intensive care unit; CCR, creatinine clearance rate; OR, odds ratio; Cl, confidence interval; TMP-SMZ, trimethoprimsulfamethoxazole.

\section{Discussion}

Although PMB is an effective drug for CR-GNB infected critical illness, the PMB-nephrotoxicity limited its use. Studies on the safety and efficacy of PMB in patients with renal diseases, such as kidney transplantation, are lacking. Here, we performed the nephrotoxicity and efficacy assessment of PMB use in renal transplant patients. We enrolled 235 renal transplant patients treated with PMB in this study. After PMB treatment, 45 patients occurred PMB nephrotoxicity, and the nephrotoxicity rate was $19.15 \%$. Among them, 44 patients were RIFLE R stage, and one patient was RIFLE I stage. The dose of PMB used in patients was $40.0(40.0-50.0) \mathrm{mg} \mathrm{q} 12 \mathrm{~h}$ with a loading dose of $41.8 \pm 9.8 \mathrm{mg}$, which is relatively lower than the international guidelines recommend dosages. Multivariate logistic regression analysis showed that ICU admission, vasoactive agents, aminoglycosides, CCR before PMB use, and mean total hospital stay were independent risk factors of PMB-nephrotoxicity in kidney transplants patients. The clinical effective rate was $97.9 \%$, and the microbiological clean rate was $66.7 \%$, which showed that PMB low dose regimens might achieve good efficacy and less nephrotoxicity.

In clinical practice, clinicians usually did not follow the recommended dose according to guidelines ${ }^{12}$ because they were worried about the effect of PMB on the renal function of transplant patients. In the real world, the impact of PMB at doses lower than recommended by the guideline on infection and renal function in kidney transplant patients are worth further exploring.

There were lacking data about the PMB use experience in renal transplant patients. A recent study about population pharmacokinetics of PMB and dosage optimization with Monte Carlo simulation found that a $75 \mathrm{mg}$ loading dose with a 50mg maintenance dose was a better option to achieve an optimal therapeutic effect (minimum inhibitory concentration $\leq 1 \mathrm{mg} / \mathrm{L}$ ) and to reduce the incidence of nephrotoxicity for patients with renal impairments. ${ }^{8}$ In a retrospective study in 92 solid organ transplant patients treated with polymyxin (polymyxins B and E), including 77 kidney transplant patients and six kidney-pancreas transplant patients, the nephrotoxicity rate was $32.6 \%$, and the duration of polymyxin was associated with significant renal dysfunction. ${ }^{9}$ Here, we found PMB low dose regimens seem to achieve good efficacy and less nephrotoxicity in renal transplant patients.

Many studies found that PMB-nephrotoxicity was associated with PMB daily dose. ${ }^{15-19}$ The administration of loading doses has increased the risk of colistin and PMB nephrotoxicity in some studies, ${ }^{16,20,21}$ but not others. ${ }^{22,23}$ Moreover, a study found that loading colistin was also independently associated with clinical failure and bacteriological failure compared with 
those who received carbapenems. ${ }^{24}$ A higher daily PMB dose ( $>30000 \mathrm{IU} / \mathrm{kg} /$ day) was independently associated with AKI. ${ }^{16}$ Trough PMB plasma concentration is an independent risk factor for its nephrotoxicity. ${ }^{7} \mathrm{~A}$ tentative target therapeutic range for plasma PMB $\mathrm{AUC}_{24}$ at a steady state of $50-100 \mathrm{mg} \cdot \mathrm{h} / \mathrm{L}$ has recently been proposed, corresponding to a plasma PMB $\mathrm{C}_{\mathrm{ss} \text {, avg }}$ range of approximately $2-4 \mathrm{mg} / \mathrm{L}$. PMB with very narrow therapeutic windows leads to difficulty selecting a safe and effective dose. ${ }^{25}$ Even in kidney transplant patients with renal insufficiency, the safe dose selection gets more challenging. In our study, the kidney transplant patients using relatively small doses compared to the international guideline, the incidence of PMB-nephrotoxicity is lower than those in previous reports. ${ }^{9,15,19}$

Multivariate logistic regression analysis showed that ICU admission, vasoactive agents, aminoglycosides, CCR before PMB use, and mean total hospital stay were independent risk factors of PMB-nephrotoxicity in kidney transplants. ICU admission patients have complex conditions, serious infections, combined with cardiac and liver dysfunction and hemodynamic instability, which may affect patients' renal insufficiency. Moreover, patients with other nephrotoxic drugs, such as aminoglycosides, may also aggravate renal insufficiency. A previous study also reported aminoglycosides as significantly associated with polymyxin nephrotoxicity. ${ }^{26}$ A study in 92 solid-organ-transplanted patients showed a statistically significant association between the duration of polymyxin treatment and significant renal dysfunction by multivariate analysis. ${ }^{9}$ Vancomycin combination with polymyxins showed significant association with renal dysfunction by univariate analysis, not in multivariate analysis. ${ }^{9}$

The clinical effective rate was $97.9 \%$, and the microbiological clean rate was $66.7 \%$. A previous study also found that after using polymyxin, adjusting dose by renal function, microbiological cure was 100\% (24 urine cultures from recipients and one blood culture from a donor, all kidney transplant patients). ${ }^{9}$ Clinical cure was $77.2 \%$, and hospital mortality was $22.8 \%$, similar to our results. ${ }^{9}$ Mean and median PMB doses employed were 922,282 and 1,000,000 IU/ day, respectively, ${ }^{9}$ similar to our results. These results imply that PMB low dose regimens can also achieve good efficacy in renal transplant patients.

Some studies focused on combining PMB with other drugs for the treatment of CR-GNB. Wasan Katip et al compared the efficacy and safety of colistin plus vancomycin to treat critically ill patients with CRAB. They found that compared with colistin alone, colistin combination with vancomycin was not necessary to manage infection caused by CRAB. ${ }^{27}$

The study also found that the addition of meropenem to colistin caused a reduction in 30-day mortality, higher clinical and microbiological responses, and did not increase nephrotoxicity compared to colistin monotherapy. ${ }^{28}$

To reduce the incidence of PMB-associated nephrotoxicity, physicians and pharmacists could collaborate on the rational use of PMB. They should cooperate in developing more precise treatment plans for patients with CR-GNB infections: appropriate PMB dosage, safe and reasonable drug combinations, and drug adjustment after nephrotoxicity. Also, they can use the TDM data of PMB to adjust medication regimens accurately.

The retrospective nature of the study imposes some limitations in our study. The time to reach an endpoint for nephrotoxicity may be underestimated because all patients lack daily creatinine measurements. Although serum urea and creatinine are easy indicators to get, they are not ideal markers for renal function assessment. TDM of PMB was not carried out in our hospital before. Therefore, the concentrations were not analyzed in this study. The single-center with limited samples also limits the quality of the article. Finally, the analysis of a population subjected to multiple interventions impacting renal function may overestimate the real nephrotoxic effect of PMB.

\section{Conclusion}

Our study found that PMB low dose regimens might achieve good efficacy and less nephrotoxicity in renal transplant patients. We should evaluate the severity of infection and renal function of patients, avoid the combined use of other nephrotoxic drugs, and minimize the course of use to reduce the occurrence of PMB-nephrotoxicity.

\section{Abbreviations}

MDR, multi-drug resistance; CR-GNB, carbapenem-resistant Gram-negative bacteria; ADRs, adverse reactions; AKI, acute kidney injury; KDIGO, Kidney Disease: Improving Global Outcomes; AKD, acute kidney disease; CKD, chronic kidney disease; SD, standard deviation; IQR, interquartile ranges; TMP-SMZ, trimethoprim/sulfamethoxazole. 


\section{Data Sharing Statement}

Available upon request to the correspondence author.

\section{Ethics Approval and Informed Consent}

The study protocol was approved by the Ethics Committees of the Second Xiangya Hospital of Central South University (LYF-2020021) in Changsha, China. This retrospective study involved patients admitted to the Second Xiangya Hospital of Central South University from 2018 to 2021.

\section{Consent for Publication}

Available.

\section{Acknowledgments}

We thank the supported grants of the Scientific Research Project of Hunan Health Commission (No. 202113010170, 202213014496). Yu-Xin Wen and Qiang Qu are co-first authors for this study.

\section{Author Contributions}

JQ conceived of this study. XLW, WML, XQT, and TTQ collected the samples. XLW, WML, and QQ performed the analysis. JQ and XLW drafted the manuscript. All authors made a significant contribution to the work reported, whether that is in the conception, study design, execution, acquisition of data, analysis and interpretation, or in all these areas; took part in drafting, revising or critically reviewing the article; gave final approval of the version to be published; have agreed on the journal to which the article has been submitted; and agree to be accountable for all aspects of the work.

\section{Funding}

This work was supported by the Scientific Research Project of Hunan Health Commission (Nos. 202113010170 and 202213014496).

\section{Disclosure}

The authors report no conflicts of interest in this work.

\section{References}

1. Vattimo Mde F, Watanabe M, da Fonseca CD, Neiva LB, Pessoa EA, Borges FT. Polymyxin B nephrotoxicity: from organ to cell damage. PLoS One. 2016;11(8):e0161057. doi:10.1371/journal.pone.0161057

2. Mohapatra SS, Dwibedy SK, Padhy I. Polymyxins, the last-resort antibiotics: mode of action, resistance emergence, and potential solutions. J Biosci. 2021;46(3):46.

3. Nation RL, Velkov T, Li J. Colistin and polymyxin B: peas in a pod, or chalk and cheese? Clin Infect Dis. 2014;59(1):88-94. doi:10.1093/cid/ ciu213

4. Sisay M, Hagos B, Edessa D, Tadiwos Y, Mekuria A. Polymyxin-induced nephrotoxicity and its predictors: a systematic review and meta-analysis of studies conducted using RIFLE criteria of acute kidney injury. Pharmacol Res. 2020;163:105328. doi:10.1016/j.phrs.2020.105328

5. Kelesidis T, Falagas ME. The safety of polymyxin antibiotics. Expert Opin Drug Saf. 2015;14(11):1687-1701. doi:10.1517/ 14740338.2015 .1088520

6. Wang P, Zhang Q, Zhu Z, et al. Comparing the population pharmacokinetics of and acute kidney injury due to Polymyxin B in Chinese patients with or without renal insufficiency. Antimicrob Agents Chemother. 2021;65(2). doi:10.1128/AAC.01900-20

7. Han L, Xu FM, Zhang XS, et al. Trough polymyxin B plasma concentration is an independent risk factor for its nephrotoxicity. Br $J$ Clin Pharmacol. 2021. doi:10.1111/bcp.15061

8. Li Y, Deng Y, Zhu ZY, et al. Population pharmacokinetics of Polymyxin B and dosage optimization in renal transplant patients. Front Pharmacol. 2021;12:727170. doi:10.3389/fphar.2021.727170

9. Mostardeiro MM, Pereira CA, Marra AR, Pestana JO, Camargo LF. Nephrotoxicity and efficacy assessment of polymyxin use in 92 transplant patients. Antimicrob Agents Chemother. 2013;57(3):1442-1446. doi:10.1128/AAC.01329-12

10. Novelli G, Morabito V, Ferretti G, et al. Safety of polymyxin-B-based hemoperfusion in kidney and liver transplant recipients. Transplant Proc. 2012;44(7):1966-1972. doi:10.1016/j.transproceed.2012.05.057

11. Kaplan B, Meier-Kriesche HU. Renal transplantation: a half century of success and the long road ahead. J Am Soc Nephrol. 2004;15 (12):3270-3271. doi:10.1097/01.ASN.0000146569.59482.8C 
12. Tsuji BT, Pogue JM, Zavascki AP, et al. International consensus guidelines for the optimal use of the polymyxins: endorsed by the American College of Clinical Pharmacy (ACCP), European Society of Clinical Microbiology and Infectious Diseases (ESCMID), Infectious Diseases Society of America (IDSA), International Society for Anti-infective Pharmacology (ISAP), Society of Critical Care Medicine (SCCM), and Society of Infectious Diseases Pharmacists (SIDP). Pharmacotherapy. 2019;39(1):10-39. doi:10.1002/phar.2209

13. Kdigo KD, Outcomes IG. Acute Kidney Injury Work Group: KDIGO clinical practice guideline for acute kidney injury. Kidney Int Suppl. 2012;2 (1): $1-138$.

14. Gibson GA, Bauer SR, Neuner EA, Bass SN, Lam SW. Influence of colistin dose on global cure in patients with bacteremia due to carbapenem-resistant gram-negative bacilli. Antimicrob Agents Chemother. 2016;60(1):431-436. doi:10.1128/AAC.01414-15

15. Zhang J, Hu Y, Shen X, Zhu X, Chen J, Dai H. Risk factors for nephrotoxicity associated with polymyxin B therapy in Chinese patients. Int J Clin Pharm. 2021;43(4):1109-1115. doi:10.1007/s11096-020-01225-8

16. Cai Y, Leck H, Tan RW, et al. Clinical experience with high-dose Polymyxin B against carbapenem-resistant gram-negative bacterial Infections-A cohort study. Antibiotics. 2020;9(8). doi:10.3390/antibiotics9080451

17. Nation RL, Rigatto MHP, Falci DR, Zavascki AP. Polymyxin acute kidney injury: dosing and other strategies to reduce toxicity. Antibiotics. 2019;8 (1). doi:10.3390/antibiotics8010024

18. Dubrovskaya Y, Prasad N, Lee Y, Esaian D, Figueroa DA, Tam VH. Risk factors for nephrotoxicity onset associated with polymyxin B therapy. J Antimicrob Chemother. 2015;70(6):1903-1907. doi:10.1093/jac/dkv014

19. Rigatto $\mathrm{MH}$, Behle TF, Falci DR, et al. Risk factors for acute kidney injury (AKI) in patients treated with polymyxin B and influence of AKI on mortality: a multicentre prospective cohort study. J Antimicrob Chemother. 2015;70(5):1552-1557. doi:10.1093/jac/dku561

20. Katip W, Uitrakul S, Oberdorfer P. Clinical outcomes and nephrotoxicity of colistin loading dose for treatment of extensively drug-resistant Acinetobacter baumannii in cancer patients. Infect Drug Resist. 2017;10:293-298. doi:10.2147/IDR.S144314

21. Rigatto MH, Oliveira MS, Perdigao-Neto LV, et al. Multicenter prospective cohort study of renal failure in patients treated with colistin versus Polymyxin B. Antimicrob Agents Chemother. 2016;60(4):2443-2449. doi:10.1128/AAC.02634-15

22. John JF, Falci DR, Rigatto MH, Oliveira RD, Kremer TG, Zavascki AP. Severe infusion-related adverse events and renal failure in patients receiving high-dose intravenous Polymyxin B. Antimicrob Agents Chemother. 2018;62(1). doi:10.1128/AAC.01617-17

23. Elefritz JL, Bauer KA, Jones C, Mangino JE, Porter K, Murphy CV. Efficacy and safety of a colistin loading dose, high-dose maintenance regimen in critically ill patients with multidrug-resistant gram-negative pneumonia. J Intensive Care Med. 2017;32(8):487-493. doi:10.1177/ 0885066616646551

24. Katip W, Yoodee J, Uitrakul S, Oberdorfer P. Efficacy of loading dose colistin versus carbapenems for treatment of extended spectrum beta lactamase producing Enterobacteriaceae. Sci Rep. 2021;11(1):18. doi:10.1038/s41598-020-78098-4

25. Lakota EA, Landersdorfer CB, Nation RL, et al. Personalizing Polymyxin B dosing using an adaptive feedback control algorithm. Antimicrob Agents Chemother. 2018;62(7). doi:10.1128/AAC.00483-18

26. Temocin F, Erdinc S, Tulek N, Demirelli M, Bulut C, Ertem G. Incidence and risk factors for colistin-associated nephrotoxicity. Jpn J Infect Dis. 2015;68(4):318-320. doi:10.7883/yoken.JJID.2014.223

27. Katip W, Oberdorfer P. Clinical efficacy and nephrotoxicity of colistin alone versus colistin plus vancomycin in critically ill patients infected with carbapenem-resistant Acinetobacter baumannii: a propensity score-matched analysis. Pharmaceutics. 2021;13(2). doi:10.3390/ pharmaceutics 13020162

28. Katip W, Uitrakul S, Oberdorfer P. A comparison of colistin versus colistin plus meropenem for the treatment of carbapenem-resistant Acinetobacter baumannii in critically ill patients: a propensity score-matched analysis. Antibiotics. 2020;9(10). doi:10.3390/antibiotics9100647

Infection and Drug Resistance

Dovepress

\section{Publish your work in this journal}

Infection and Drug Resistance is an international, peer-reviewed open-access journal that focuses on the optimal treatment of infection (bacterial, fungal and viral) and the development and institution of preventive strategies to minimize the development and spread of resistance. The journal is specifically concerned with the epidemiology of antibiotic resistance and the mechanisms of resistance development and diffusion in both hospitals and the community. The manuscript management system is completely online and includes a very quick and fair peer-review system, which is all easy to use. Visit http://www.dovepress.com/testimonials.php to read real quotes from published authors.

Submit your manuscript here: https://www.dovepress.com/infection-and-drug-resistance-journal 